\title{
Kernos
}

Revue internationale et pluridisciplinaire de religion grecque antique

29 | 2016

Varia

\section{Hermes, lo scettro, l'ariete. Configurazioni mitiche della regalità nella Grecia antica}

\section{Dominique Jaillard}

\section{(2) OpenEdition \\ Journals}

\section{Édition électronique}

URL : http://journals.openedition.org/kernos/2420

DOI : 10.4000/kernos.2420

ISSN : 2034-7871

\section{Éditeur}

Centre international d'étude de la religion grecque antique

\section{Édition imprimée}

Date de publication : 1 octobre 2016

Pagination : 429-431

ISSN : 0776-3824

\section{Référence électronique}

Dominique Jaillard, « Hermes, lo scettro, l'ariete. Configurazioni mitiche della regalità nella Grecia antica », Kernos [En ligne], 29 | 2016, mis en ligne le 01 octobre 2016, consulté le 17 novembre 2020 URL : http://journals.openedition.org/kernos/2420 ; DOI : https://doi.org/10.4000/kernos.2420

Ce document a été généré automatiquement le 17 novembre 2020.

Kernos 


\title{
Hermes, lo scettro, l'ariete. Configurazioni mitiche della regalità nella Grecia antica
}

\author{
Dominique Jaillard
}

\section{RÉFÉRENCE}

Carmine PISANO, Hermes, lo scettro, l'ariete. Configurazioni mitiche della regalità nella Grecia antica, Napoli, M. D'Auria editore, 2014.1 vol. $15 \times 21 \mathrm{~cm}, 299$ p. (Religioni e storia, 3). ISBN : 978-88-7092-362-9.

Issu de sa thèse de doctorat, l'Hermès de Carmine Pisano (C.P.) est un livre important, élégamment écrit, qui fera date dans l'exploration des configurations grecques de la "souveraineté ", et une contribution majeure à la compréhension des polythéismes antiques. L'ouverture, un commentaire du passage d'Hérodote (III, 38) mettant en scène l'enquête ethnographique conduite par Darius sur les coutumes funéraires et les formes de piété inconciliables des Grecs et des Indiens donne d'emblée le ton - esprit et méthode. L'antiquité sera traitée comme un territoire des écarts, objet d'une enquête anthropologique pour laquelle les sources textuelles dont dispose «l'observateur moderne » constituent indissolublement « la 'barrière' qui empêche de voir de manière distincte et directe la 'réalité' des savoirs partagés » et le seul « fil d'Ariane " possible laissant «entrevoir» les représentations culturelles affleurant dans ces moments privilégiés du processus de communication où «il devient nécessaire d'user de la langue pour parler de la langue, dans l'espace de la communication métalinguistique » (p. 14). Pour engager à nouveaux frais «l'étude des systèmes de représentation de la royauté en Grèce ancienne ", l'A. a fait un choix dont chaque chapitre du livre confirmera la pertinence heuristique : «définir les mécanismes de construction de la légitimité et de l'autorité de la figure royale en se fondant sur l'étude du réseau complexe de relations qui lie la sphère de la souveraineté à Hermès, le dieu qui transmet aux rois les insignes du pouvoir, sceptre et bélier d'or» (p.16). Pour 
reprendre la formule - image de M. Detienne, - C.P. a trouvé là deux excellents "réactifs" pour explorer les paysages de la basileia à partir d'une puissance qui apparaît à la fois à ses marges, tenu à distance - l'accès à la boulé de Zeus lui est refusé dans l'Hymne homérique - et en son cœur, figurant tantôt en simple serviteur, tantôt investi de pouvoirs redoutables, mais opérateur discret assumant les liaisons sans lesquelles royauté et souveraineté se dissolvent. De cette ambivalence efficace que nous avions exploré dans le champ du sacrifice et de la parole, C.P. dégage tous les enjeux pour l'analyse des agencements grecs de la souveraineté.

2 Kerux ou anax? En terme d'alternative, l'opposition entre un Hermès héraut ou messager, «dans la tradition grecque, Hermès est tout sauf un dieu souverain » (p. 23), et un Hermès anax, roi, attesté dans un passage de l'Iliade (II, 104) racontant la transmission du sceptre de Zeus à Pélops et à sa descendance, est un produit de la philologie et de l'historiographie modernes. Alors que pour les scholiastes anciens, le sceptre qu'Hermès Argeiphontes reçoit de Zeus fonctionne comme le bâton du héraut, objet présentifiant un pouvoir souverain, les savants modernes (notamment F. Cassola, G. Kirk et J. Orgogozo) ont majoritairement repéré une contradiction aisément interprétable en terme de survivance d'une fonction souveraine qu'Hermès aurait assumée à l'époque mycénienne. C.P. fait justice de cette hypothèse, mais ce n'est peutêtre pas là le plus important. À l'occasion d'un détour historiographique, l'A. déroule un des fils d'Ariane les plus féconds de sa recherche, l'exploration des positions d'Hermès kerux, des relations de sa charge de héraut tant avec d'autres modalités de sa propre action qu'avec la royauté de Zeus. Ainsi l'ouvrage dessine-t-il en filigrane le réseau complexe des fonctions et significations attachées au héraut - humain ou divin -, une configuration héraldique originale dont la cohérence tend autrement à échapper. La leçon est de méthode, où une étude prioritairement centrée sur de la «mythologie » jette une lumière vive sur une fonction sociale que la projection de nos catégories modernes tend à défaire en en brouillant les contours. Car c'est aussi une des forces du livre de C.P. de proposer un parcours qui fait passer le lecteur d'une "reconstruction "étique" (de l'extérieur) des rapports d'Hermès avec le pouvoir royal à une enquête "émique" (...) soumettant aux autochtones hellénophones les résultats précédemment obtenus » (p. 15).

3 L'architecture du livre en dépend. Aux deux premières parties « Hermès, le sceptre et la parole d'autorité », «Hermès, le bélier et la légitimité du pouvoir » confrontant le dieu aux « réactifs » du sceptre et du bélier, de manière à faire émerger des savoirs partagés qui resteraient autrement largement implicites, répond une troisième partie centrée sur la question de l'hermeneia et sur les interpretationes dont le dieu est l'objet (mais aussi, par ses fonctions propres, l'auteur). C.P. analyse les processus de traduction et d'identification d'un dieu étranger à Hermès comme une contre-épreuve à son étude des fonctions de l'Hermès kerux, porte parole et interprète (hermeneus) de Zeus, une sorte de «test » d'autant plus exigeant qu'il met en jeu des contextes marqués d'une dimension d'altérité religieuse forte, judaïque ou chrétienne - du Moïse herméneute des lettres sacrées (hiera grammata) d'Artapan à l'assimilation que les Actes des apôtres attribuent aux habitants de Lystre de Barnabé à Zeus et de Paul à Hermès "parce que c'est lui qui conduisait le discours (hegoumenos tou logou)». Des ensembles de représentations partagées, des langues communes s'en dégagent, sur le fond desquels les usages pragmatiques dont témoignent les sources textuelles opèrent sélections, resémentisations, réorientations. Tout en constituant un moment décisif de la mise à 
l'épreuve de sa méthode par l'A., cette troisième partie peut aussi se lire pour ellemême, notamment le chapitre "Traduction et signifiés émiques" qui interroge les processus antiques de traduction comme modalités de connaissance et revient longuement sur quelques lectures de l'hermeneia et de l'interpretatio, de Platon à Dumézil, de Rudhardt à Bettini. Il faut aussi souligner la finesse des analyses consacrées aux relations - différences entre hermeneia et exegesis.

C'est assez dire que la richesse du livre est telle qu'il faudrait pouvoir rendre compte de ses analyses dans tous leurs détails. Dans le cadre de cette présentation, nous nous contenterons de mettre en exergue quelques moments forts et novateurs. Mentionnons la mise en évidence du statut éminent d'un certain nombre de figures de l'entourage royal, le kerux au premier chef, désignées, dans le rapport hiérarchique les liant au souverain, par des termes en faisant des « serviteurs ", alors que leur relation au roi en fait d'abord les détenteurs et acteurs à distance d'un aspect fondamental d'une puissance royale diffractée, une part intégrante de la basileia en acte. Une différence très claire est tracée entre l'angelos et le kerux, entre Iris répétant littéralement le message dont elle est chargée et Hermès interprète inventant et déployant la rhétorique et les puissances de persuasion appropriées à la situation, parole mobile faisant cheminer et donnant effet à la boulé royale. Signalons aussi la mise en évidence des homologies entre skeptron et rhabdos (au risque peut-être de gommer des différences qu'il faudrait réinterroger), pouvoir de la rhabdos de "réaliser, de rendre efficace " les thesphata de Zeus / du roi (p. 106), en lien à un accord (symbolon) et à la parole qui le porte (dans un très beau commentaire de la fin de l'Hymne homérique à Hermès, p. 106) ou l'étude minutieuse des délibérations des basileis réunis autour d'Agamemnon dans l'Iliade pour lesquelles le sceptre fonctionne "comme la trace visible d'un réseau invisible de relations » (p. 133), impliquant des modalités d'action et d'expression de la puissance dont le langage du polythéisme analyse, dissèque et articule les composantes et les moments. Dieux explicitement nommés, objets - instruments comme la rhabdos, le sceptre ou la lyre, kudos (sur lequel l'auteur revient un peu vite) y apparaissent au même degré comme des instances constitutives du panthéon.

5 Alors que la première partie prend pour fil l'exploration des relations entre Hermès, sceptre et "autorité », la seconde, en rupture avec les interprétations longtemps dominantes - dont l'A. fait ressortir les présupposés anthropologiques, notamment les théories de la royauté magique - redéfinit l'agneau ou bélier d'or comme puissance de légitimation en procédant à une analyse comparative des mythes argien et argonautique.

6 Le livre de C.P. contribue ainsi, en creux, au travail de déconstruction des concepts modernes, monolithiques, de la souveraineté, forgés à partir d'éléments de philosophie grecque et de modèles romains (non sans contresens) et, pour nous, obstacle épistémologique majeur à la compréhension des paysages grecs de la basileia. L'exploration de ces derniers exige au contraire de repérer les articulations fines d'un champ d'action distribué entre des acteurs multiples dont la figure du roi, pour centrale qu'elle puisse être donnée, n'est qu'une des positions solidaires. Comme contre épreuve, l'étude des positions quelquefois si discrètes et néanmoins si invasives que Zeus tend à occuper dans les panthéons des cités, s'impose. Sans que l'auteur y insiste, le livre illustre aussi qu' "un dieu», en régime polythéiste grec, ne peut jamais être appréhendé que comme un mini-panthéon; en chacune des positions qu'il occupe, il n'est qu'une partie de « lui-même » incluant dans le paquet de relations qui le constitue 
bien des aspects des « autres ». Avec l'Hermès de C. Pisano, c'est tout un segment « du » panthéon grec qui s'éclaire.

\section{AUTEURS}

DOMINIQUE JAILLARD

Université de Genève 